\section{Reanimación cardiopulmonar en pacientes con enfermedad por COVID-19 en el ambiente hospitalario}

\author{
Cardiopulmonary resuscitation in patients with \\ COVID-19 in the hospital environment
}

\author{
Dr. Enrique Monares-Zepeda,* Dr. Job E Rodríguez-Guillén, ${ }^{\ddagger}$ \\ Dr. Rafael Eduardo Herrera-Elizalde,,$^{\S}$ Dr. Sebastián Ugarte-Ubiergo," \\ Dr. Arturo Garza-de la Maza"
}

\begin{abstract}
RESUMEN. La pandemia COVID-19 nos ha obligado a replantearnos la manera en la que practicamos algunos aspectos de la medicina. La reanimación cardiopulmonar es una práctica que genera partículas en aerosol provenientes de la vía aérea, lo cual incrementa el riesgo de contagio por SARS-CoV-2. En esta revisión se consultan las recomendaciones internacionales sobre el tema, se definen momentos de alto riesgo y se establecen recomendaciones sobre compresiones, ventilación, terapia eléctrica e incluso farmacológica en pacientes con paro cardíaco y con diagnóstico de COVID-19, buscando no sólo el bienestar del paciente, sino también la seguridad del personal de salud.
\end{abstract}

ABSTRACT. The COVID-19 pandemic has forced us to rethink the way we practice some aspects of medicine. Cardiopulmonary resuscitation is a practice that generates aerosol particles from the airway, which increases the risk of SARS-CoV-2 infection. In this review, the international recommendations on the subject are consulted, high-risk moments are defined and recommendations are established on compressions, ventilation, electrical therapy and even pharmacology in patients with cardiac arrest and with a diagnosis of COVID-19, seeking not only the well-being of the patient, but also the safety of health personnel.

Primum Non Nocere, en especial a nosotros mismos y nuestro equipo.

$\mathbf{N}$ ota de los autores: todas las recomendaciones aquí vertidas tienen un muy bajo nivel de evidencia y son más un punto de partida para tratar de encontrar lo que es correcto, no nos declaramos poseedores de una verdad que nadie tiene. La única respuesta correcta en estos momentos es: aún no sabemos. Estamos ante el primer escalón de la búsqueda, hemos dado el primer paso y eso es todo.

Los organismos internacionales sobre reanimación han emitido recomendaciones sobre la conducta a tomar en este escenario ${ }^{(1,2)}$. $\mathrm{Al}$ ser considerado un procedimiento generador de partículas en aerosol ${ }^{(3)}$, establecen lo siguiente:

- Recomendamos en cada ingreso determinar si existe o no orden de no intentar reanimación mediante una opinión informada con el paciente si es posible o familiares, insistiendo en las posibilidades de éxito ${ }^{(1)}$.

- Recomendamos establecer equipos de respuesta rápida para detectar casos evitables de paro cardíaco mediante las escalas de respuesta rápida ${ }^{(4)}$ (Tablas 1 y 2).

- Su práctica se debe realizar en habitaciones aisladas y con equipo de protección personal (como mínimo Mascarilla N95 o FFP3, protectores para ojos Z87.1, careta plástica o su equivalente y guantes) $)^{(1,3)}$.

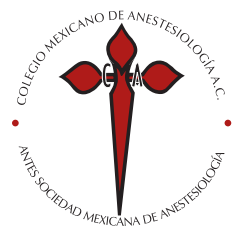

Palabras clave: COVID-19, paro cardíaco, reanimación cardiopulmonar, prono, desfibrilación, ventilación, QT prolongado, equipo de protección personal, arritmias.

Keywords:

COVID-19, cardiac arrest, cardiopulmonary resuscitation, prone, defibrillation, ventilation, prolonged QT, personal protective equipment, arrhythmias.

\footnotetext{
* Médico Intensivista, Centro Médico ABC, CDMX.

‡ Médico Urgenciólogo y de Medicina Crítica, Jefe de Urgencias Hospital H más Querétaro. $\S$ Anestesiólogo Cardiovascular Pediátrico, adscrito al Servicio de Anestesiología, Instituto Nacional de Cardiología «Ignacio Chávez», Cuidad de México, México.

" Médico Intensivista, Jefe del Departamento de Terapia Intensiva INDISA Clinic, Universidad Andrés Bello, Santiago de Chile, Chile. " Médico Urgenciólogo e Intensivista. Residente de Cuidados Críticos Cardiovasculares, Instituto Nacional de Cardiología «Dr. Ignacio Chávez» Ciudad de México, México.

Solicitud de sobretiros: Dr. Enrique Monares Zepeda Centro Médico ABC. Ciudad de México.

Medicina Crítica y Terapia Intensiva. E-mail:

enrique_monares@hotmail.com
}

Recibido para publicación: 02-04-2020

Aceptado para publicación: 09-04-2020 
- El mínimo personal necesario.

- Antes de iniciar la reanimación cardiopulmonar (RCP), la vía aérea del paciente debe ser aislada, ya sea utilizando una mascarilla facial o un dispositivo supraglótico conectado a filtro viral (Figura 1), o bien, si está intubado, conectarlo al circuito del ventilador asegurando evitar la desconexión ${ }^{(1)}$.

Recomendamos no romper el protocolo de aislamiento de pacientes en ningún momento de la reanimación ${ }^{(3)}$.

Recomendamos RCP de alta calidad. Compresiones de 100 a 120 por minuto, compresión torácica adecuada (5 a 6 cm) con suficiente reexpansión (tanto en desplazamiento torácico como en velocidad) y mínimas interrupciones ${ }^{(5)}$.

Recomendamos aislar la vía aérea, evitando ventilación bolsa máscara reservorio o cualquier otro sistema abierto de ventilación ${ }^{(6)}$.

Recomendamos en el contexto de COVID-19, la desfibrilación inmediata (en los primeros 60 segundos) para ritmos meritorios de esta terapia por sobre el inicio de compresiones, ya que ofrece la posibilidad al paciente de ser resucitado disminuyendo el riesgo de contagio (Algoritmo 1) $^{(2)}$.

Para ritmos tales como asistolia y actividad eléctrica sin pulso, la RCP de alta calidad con vía aérea aislada sin intentos de ventilación, el apoyo farmacológico cardiovascular, así como la búsqueda de la etiología. Sólo se brindará reanimación si el personal cuenta con equipos de protección personal a partículas en el momento del evento de colapso cardiovascular (Algoritmo 1)(2).

No recomendamos iniciar esfuerzos de reanimación sin protección adecuada. ${ }^{(3)}$

Transcurridos los dos primeros minutos en pacientes donde la etiología del evento esté relacionado a hipoxemia y sea necesaria la manipulación de la vía aérea, la debe realizar el personal con la mayor experiencia en el tema. Se recomienda la intubación orotraqueal directa (idealmente con videolaringoscopio) asistida con Bouguie ${ }^{(7)}$, minimizando el tiempo de

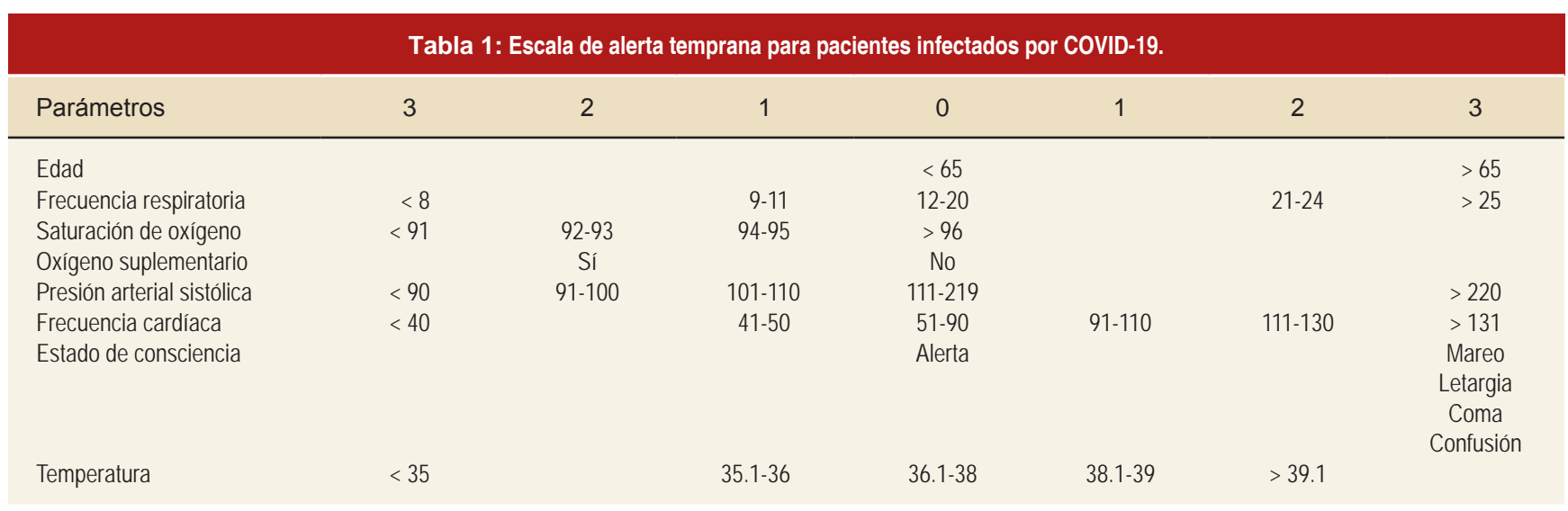

Tabla 2: Reglas y puntaje para alerta temprana para pacientes infectados por COVID-19.

\begin{tabular}{|c|c|c|c|c|c|}
\hline Puntaje & $\begin{array}{l}\text { Nivel de } \\
\text { riesgo }\end{array}$ & $\begin{array}{l}\text { Nivel de } \\
\text { alerta }\end{array}$ & $\begin{array}{l}\text { Frecuencia de } \\
\text { monitorización }\end{array}$ & Respuesta clínica & Solución \\
\hline 0 & 1 & & Cada 12 horas & Monitoreo de rutina & 1 \\
\hline $1-4$ & Bajo & Amarillo & Cada 6 horas & $\begin{array}{l}\text { Evaluación en la cama del paciente por } \\
\text { enfermería }\end{array}$ & $\begin{array}{l}\text { Monitoreo convencional/incrementar } \\
\text { frecuencia de monitoreo/informar médicos }\end{array}$ \\
\hline $\begin{array}{l}\text { 5-6 o } 3 \text { en un } \\
\text { parámetro }\end{array}$ & Medio & Naranja & Cada 1 a 2 horas & $\begin{array}{l}\text { Evaluación por enfermería con notificación } \\
\text { a médico }\end{array}$ & $\begin{array}{l}\text { Mantener tratamiento existente/ajustar } \\
\text { manejo del dolor/disponibilidad de los ERR }\end{array}$ \\
\hline$>7$ & Alto & Rojo & Continuo & $\begin{array}{l}\text { Evaluación por enfermería con notificación } \\
\text { a médico/monitorización por el ERR }\end{array}$ & Manejo por ERR \\
\hline$>7$ & Alto & Negro & Continuo & \multicolumn{2}{|c|}{$\begin{array}{l}\text { - Los pacientes se encuentran en una fase final e irreversible de su enfermedad } \\
\text { enfrentando inminente muerte, como lesión cerebral severa, falla orgánica múltiple, } \\
\text { falla hepática crónica en fase terminal, enfermedad pulmonar severa en fase } \\
\text { terminal, cáncer metastásico } \\
\text { - Se debe definir con un grupo de expertos la admisión al hospital }\end{array}$} \\
\hline
\end{tabular}

ERR = equipos de respuesta rápida. 
intubación, con las barreras de protección disponibles, y evitar realizar ventilación con presión positiva con mascarilla facial (Algoritmo 2) $)^{(1,2)}$.

Los retos relacionados a la atención pulmonar de este tipo de pacientes, nos han llevado a iniciar protocolos de

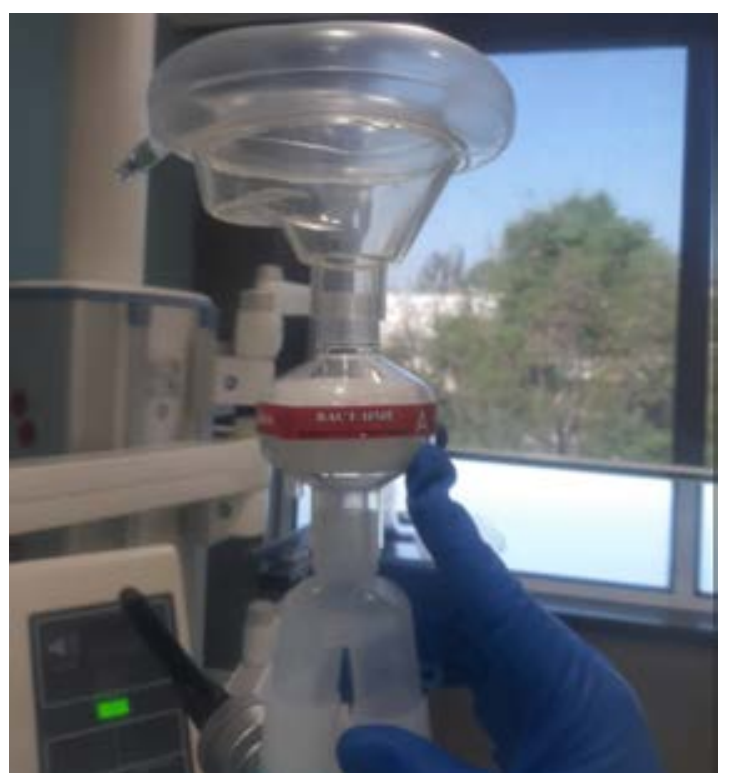

Figura 1: Aislamiento de vía aérea con circuito cerrado, filtro y altos flujos de $\mathrm{O}_{2}$. ventilación mecánica en prono(6), voltear al paciente de forma emergente implica el esfuerzo de múltiples elementos de la salud, con la potencial exposición al patógeno en tal maniobra. Realizar RCP en prono es una práctica con algún grado de recomendación y éxito en otros contextos, colocando las manos sobre la línea media a la altura de las escápulas, durante un ciclo de reanimación de 2 minutos; en caso de no ser efectiva, se recomienda voltear al paciente, contraindicado en este contexto ${ }^{(8)}$. Si existieran ritmos desfibrilables, se puede realizar desfibrilación en prono, colocando la pala de ápex lateral izquierdo y la pala esternón en la región paravertebral derecho, con máxima energía posible ${ }^{(9)}$, considerando el esquema de tres descargas consecutivas ${ }^{(10)}$, esta recomendación tiene un grado de evidencia muy bajo y su único sustento es evitar al máximo la exposición del personal de salud (Figura 2).

En resumen, las recomendaciones concretas para la atención del paro cardíaco en paciente con SARS-CoV-2 son las siguientes:

- Individualizar cada caso estableciendo orden o no de reanimación desde el ingreso.

- Recomendamos establecer equipos de respuesta rápida para detectar casos en deterioro para prevenir eventos de paro (Algoritmo 3) (Tablas 1 y 2)(4).

\section{PARO CARDÍACO (PC) Y COVID-19}

Individualizar casos, definir orden de NO reanimación desde su ingreso

Prevención: Equipos de Respuesta Rápida ante deterioro de pacientes

Cualquier esfuerzo de Reanimación debe realizarse con

\section{Equipo de Protección Personal (EPP)}

\section{Fibrilación Ventricular y \\ Taquicardia Ventricular}

- Desfibrilación Inmediata, 3 dosis secuenciales sin realizar RCP

- Único Procedimiento que se podrá Realizar sin EPP.

- Aplicable en Pacientes en Supino o Prono

\section{Situaciones Especiales}

- Decúbito Prono: Desfibrilación Inmediata y RCP sólo compresiones son aceptables. No recomendamos voltear al paciente

- QTe Prolongado: Coadyuvar la RCP con dosis de Lípidos al $20 \%, 100 \mathrm{ml}$ Intravenoso
Asistolia y

Actividad Eléctrica Sin Pulso

- Durante los Primeros 2 minutos de PC. Aislar Via Aérea y realizar RCP sólo con las manos.

- Siguiente Ciclo, control de Vía Aérea y ventilación con circuito cerrado. Verificar Intubación Orotraqueal via Capnografia

\section{Momentos Críticos con Riesgo de PC}

- Paro respiratorio por deterioro en pacientes no intubados

- Tormenta eléctrica por miocarditis

- Paro durante manejo de via aérea

- Prolongación de QTe (relacionado a fármacos y otros)

- Pérdida de la via aérea, especialmente en prono

- Deterioro respiratorio bajo ventilación mecínica
Figura 2:

Resumen de recomendaciones. 

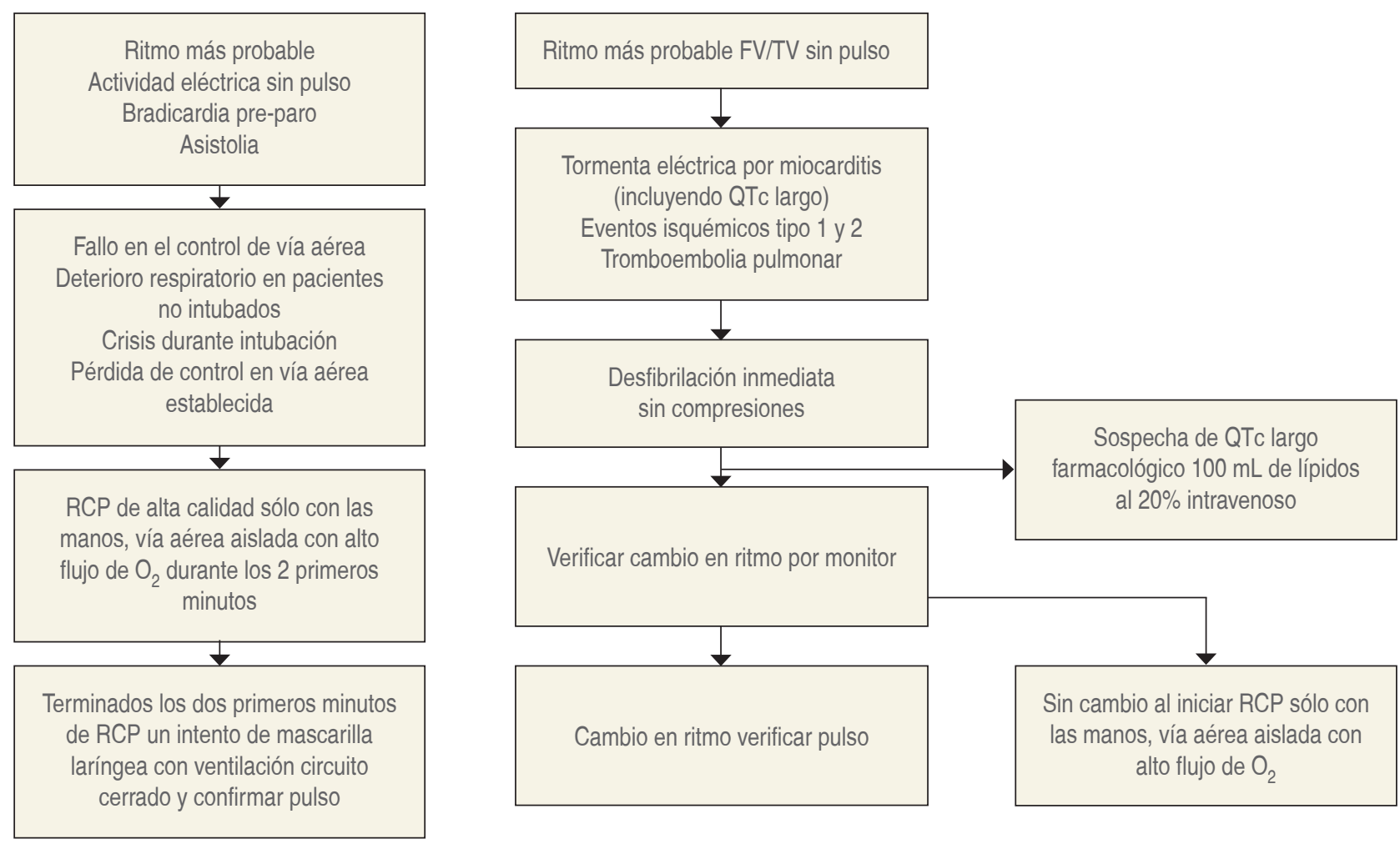

Algoritmo 1: Manejo acorde a ritmo inicial de paro cardíaco y situaciones especiales.

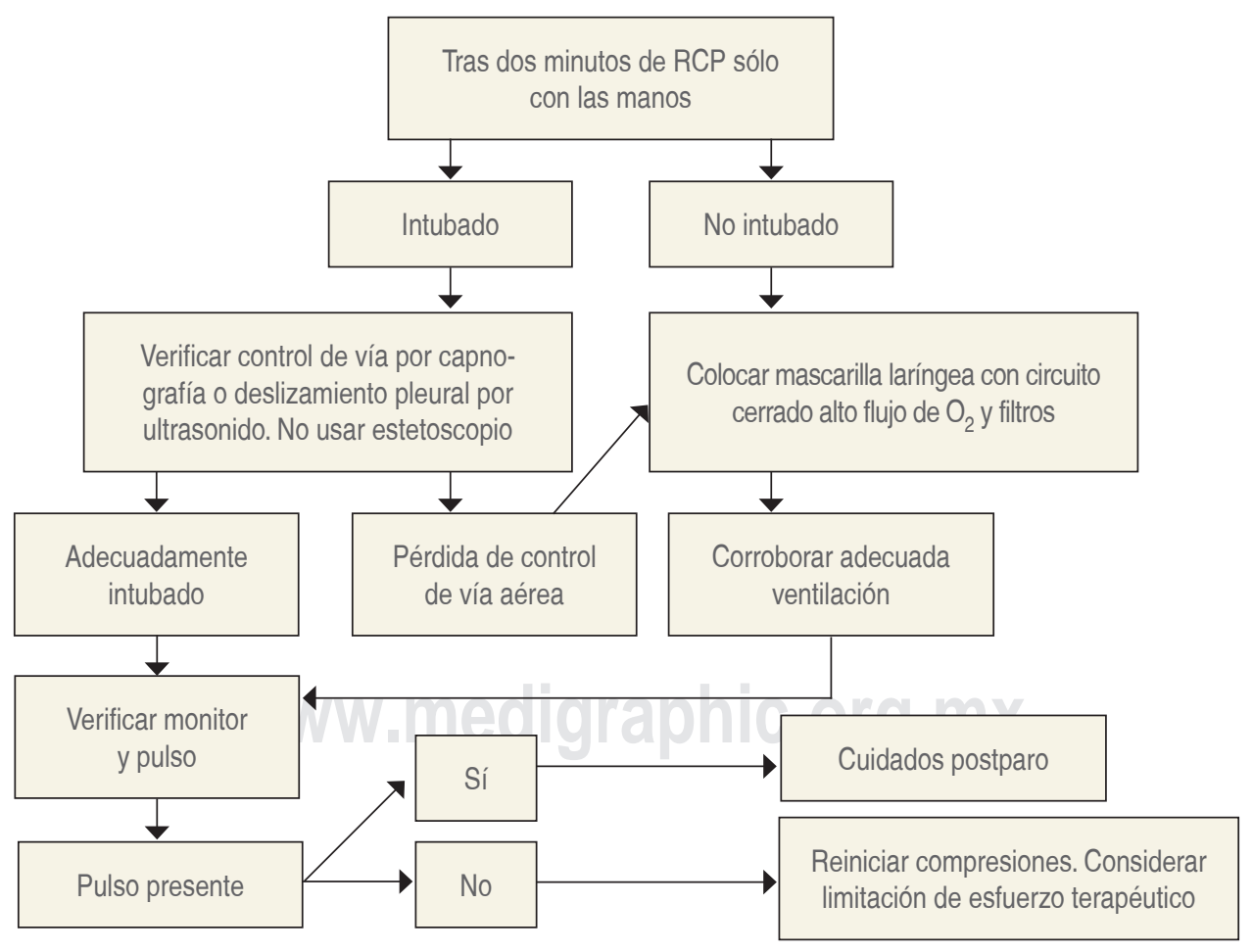

Algoritmo 2: Recomendaciones durante el manejo de vía aérea. 


\section{ALGORITMOS PARA ATENCIÓN DE PARO CARDÍACO Y COVID-19}

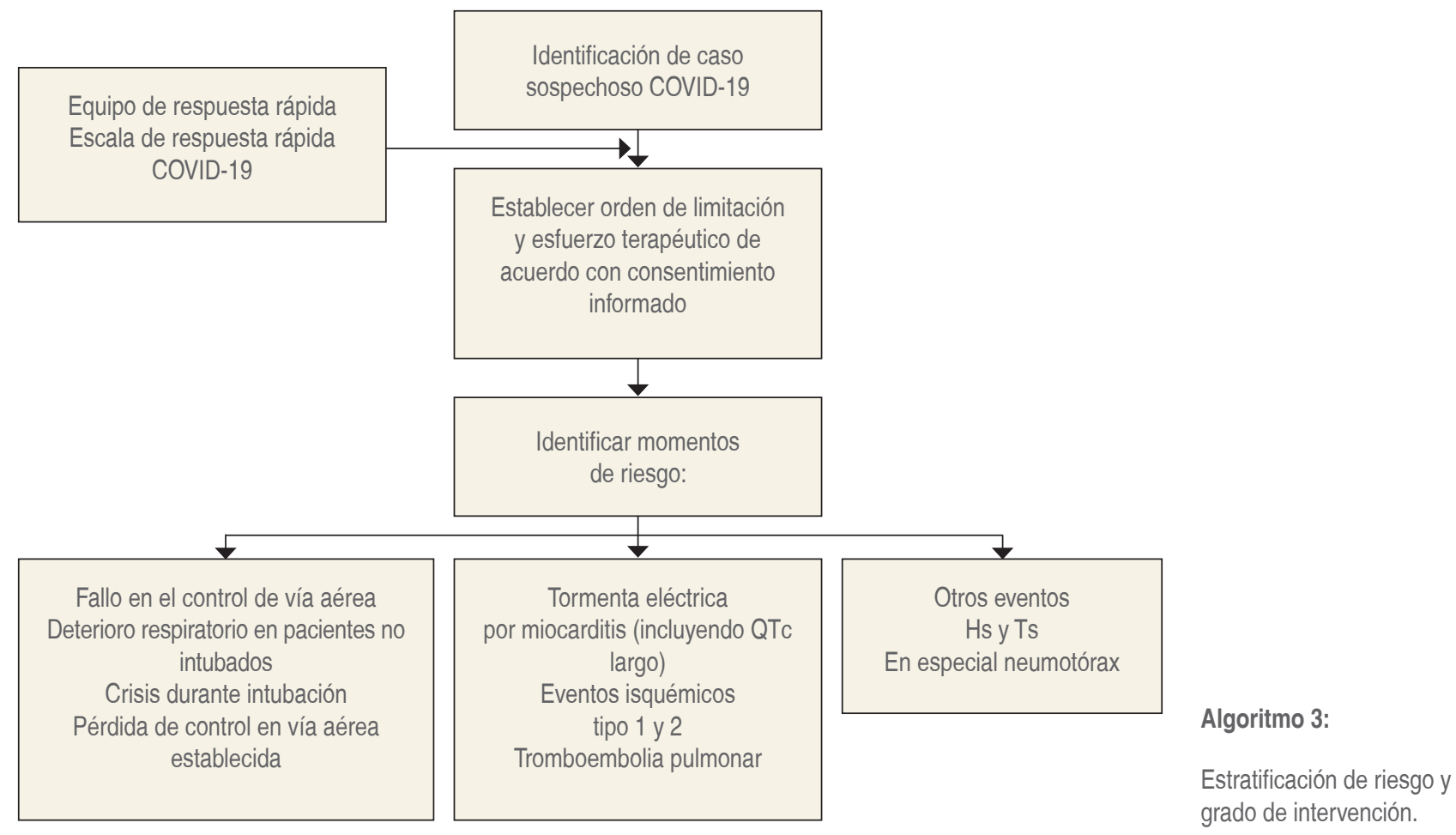

- Recomendamos tener los momentos críticos de riesgo de paro cardíaco (Algoritmo 3).

- Paro respiratorio por deterioro en pacientes no intubados.

- Tormenta eléctrica por miocarditis.

- Paro durante manejo de vía aérea.

- Prolongación de QTc (relacionado a fármacos y otros).

- Pérdida de la vía aérea, especialmente en prono.

- Deterioro respiratorio bajo ventilación mecánica.

- Poner en balanza el riesgo/beneficio, la exposición del personal de salud al patógeno no es una opción aceptable bajo ningún contexto.

- Se debería practicar RCP de alta calidad cuando se cuente con equipo de protección personal para este patógeno en el momento del evento de paro cardíaco, y sólo podrán participar en la reanimación aquéllos que cuenten con ese requisito.

- Recomendamos aislamiento de la vía aérea y RCP de alta calidad sólo con las manos los 2 primeros minutos del evento.

- Recomendamos en escenarios de FV o TV sin pulso, la desfibrilación inmediata, en tres dosis de forma secuencial sin practicar compresiones torácicas (Algoritmo 1).

- Único procedimiento que se puede realizar sin equipo de protección personal.

- Esta recomendación puede realizarse en posición supina o prono.
- En pacientes con asistolia o actividad eléctrica sin pulso, sólo persona con equipo de protección personal completo podría participar en los esfuerzos de reanimación, con el mínimo personal necesario. Durante el primer ciclo se realizarán sólo compresiones asegurando el aislamiento de la vía aérea. En el segundo ciclo se hará control de la vía aérea y ventilación mediante circuito cerrado. En caso de previo control de vía aérea hay que corroborar adecuada posición del tubo, recomendamos un solo evento de laringoscopía para colocación o confirmación del tubo endotraqueal y corroboración mediante capnografía. No recomendamos corroboración a través de estetoscopio, debido a los riesgos de contaminación (Algoritmo 1).

- La desfibrilación temprana, e incluso un ciclo de 2 minutos de RCP, son aceptables en decúbito prono. No recomendamos cambiar de posición al paciente a decúbito supino (girarlo) por el riesgo de exposición.

- En caso de paro por arritmias secundarias a prolongación de QTc corregido por fármacos, recomendamos una dosis de $100 \mathrm{~mL}$ de lípidos al 20\%. Recomendamos medir constantemente el intervalo QTc, especialmente si se emplean más de dos drogas con riesgo de prolongación de QT (azitromicina, hidroxicloroquina, Kaletra, etcétera) ${ }^{(11)}$. 


\section{REFERENCIAS}

1. AHA. Heart. Interim guidance to reduce COVID-19 transmission during resuscitation care [press release]. 19 March 2020. Available in: https://newsroom.heart.org/news/interim-guidance-to-reduce-covid-19transmission-during-resuscitation-care. 1 April 2020.

2. UK Resuscitation Council Guidance. Resuscitation Council (UK) Statement on COVID-19 (coronavirus) CPR and Resuscitation. March 2020. Available in: https://www.resus.org.uk/media/statements/ resuscitation-council-uk-statements-on-covid-19-coronavirus-cpr-andresuscitation/. 1 April 2020.

3. CDC. Centers for Disease Control and Prevention. Interim Infection Prevention and Control Recommendations for Patients with Suspected or Confirmed Coronavirus Disease 2019 (COVID-19) in Healthcare Settings. 19 Marzo 2020. Available in: https://www.cdc.gov/coronavirus/2019ncov/infection-control/control-recommendations.html?CDC_AA_ refVal=https\%3A\%2F\%2Fwww.cdc.gov\%2Fcoronavirus\%2F2019ncov\%2Fhcp\%2Finfection-control.html\#take_precautions. 1 April 2020.

4. Xuelian Liao, Bo Wang, Yan Kang. Novel coronavirus infection during the 2019-2020 epidemic: preparing Intensive Care Units-the experience in Sichuan Province, China. Intensive Care Med. 2020;46:357-360.

5. Kleinman ME. Part 5: Adult Basic Life Support and Cardiopulmonary Resuscitation Quality, 2015 American Heart Association Guidelines
Update for Cardiopulmonary Resuscitation and Emergency Cardiovascular Care. Circulation. 2015;132:S414-S435.

6. WHO Int. World Health Organization. Clinical management of severe acute respiratory infection (SARI)'when COVID-19 disease is suspected interim. 2020. Available in: https://www.who.int/docs/default-source/ coronaviruse/clinical-management-of-novel-cov.pdf?sfvrsn=bc7da51 7 10\&download. 1 April 2020.

7. Brewster DJ. Med J Aust. Consensus statement: Safe Airway Society principles of airway management and tracheal intubation specific to the COVID-19 adult patient group. 16 March 2020. Available in: https:// www.mja.com.au/journal/2020/consensus-statement-safe-airwaysociety-principles-airway-management-and-tracheal. 1 April 2020.

8. Bhatnagar V. Cardiopulmonary resuscitation: unusual techniques for unusual situations. J Emerg Trauma Shock. 2018;11:31-37.

9. Nanjangud P. Cardiopulmonary resuscitation in adult patients in prone position. Indian J Respir Care. 2017;6:791-792.

10. Dunning J. Resuscitation of patients who arrest after cardiac surgery. Ann Thorac Surg. 2017;103:1005-1020.

11. Ten Broeke R, Mestrom E, Woo L, Kreeftenberg H. Early treatment with intravenous lipid emulsion in a potentially lethal hydroxychloroquine intoxication. Neth J Med. 2016;74:210-214 